\title{
Critical bubbles and implications for critical black strings
}

\author{
Olivier Sarbach \\ Department of Mathematics and Department of Physics and Astronomy, \\ Louisiana State University, 202 Nicholson Hall, Baton Rouge, Louisiana 70803-4001, USA \\ Luis Lehner \\ Department of Physics and Astronomy, Louisiana State University, \\ 202 Nicholson Hall, Baton Rouge, Louisiana 70803-4001, USA
}

\begin{abstract}
We demonstrate the existence of gravitational critical phenomena in higher dimensional electrovac bubble spacetimes. To this end, we study linear fluctuations about families of static, homogeneous spherically symmetric bubble spacetimes in Kaluza-Klein theories coupled to a Maxwell field. We prove that these solutions are linearly unstable and posses a unique unstable mode. We further show that the associated growth rate depends only on the mass per unit length of the background solution. Additionally, by a double analytical continuation this mode can be seen to correspond to marginally stable stationary modes of perturbed black strings whose periods are integer multiples of the Gregory-Laflamme critical length. This allow us to rederive recent results about the behavior of the critical mass for large dimensions and to generalize them to charged black string cases.
\end{abstract}

PACS numbers: 11.25.Sq, 04.50.+h, 04.25.-g

\section{INTRODUCTION}

Bubble spacetimes were originally studied for, among other reasons, their connection with negative energy solutions 1, 2, 3]. The behavior of bubble spacetimes has been the subject of new scrutiny and use in recent years. Among these, their use in defining new solutions of Kaluza-Klein black holes [4, 5, 6, 7], studies of possible phases of KaluzaKlein black holes [7], solutions in inflationary universe in five-dimensional spacetimes [8] and their dynamical behavior under different interactions via numerical simulations $[9,10]$.

In particular, the numerical simulations presented in Ref. 9, 10 reveal strong indications of the possible existence of critical phenomena in bubble spacetimes. That is, there exists a critical value $k=k_{c}$ in the parameter specifying the strength of the $\mathbf{U}(\mathbf{1})$ gauge field in the initial data for the bubble. For this value, it was observed that, after some transient behavior, the solution approaches a static bubble configuration which we call the critical bubble solution. For values of $k$ close to $k_{c}$ the solution approaches the critical bubble and stays close to it for some time and then either disperses away or collapses to a black string. The amount of proper time $\tau$ that the solution remains close to the static one obeys the relation $\tau / R_{0} \simeq-\Gamma \ln \left(\left|k-k_{c}\right|\right)$, where $R_{0}$ is the bubble location -which is fixed by the ADM mass per unit length- and $\Gamma$ is a universal parameter that does not depend on the initial data.

In the present work we put on firm grounds such observation by studying linear perturbations off the critical solutions in a $q+3$ dimensional spacetime endowed with $\mathbf{U}(\mathbf{1}) \times \mathbf{S O}(\mathbf{q}+\mathbf{1})$ symmetry and elucidating the existence of a unique unstable exponential in time growing mode. Furthermore, we show that the growth rate $\Omega / R_{0}$ associated to this instability has the property that $\Omega$ does not depend on the family of critical solutions and hence explains the universal behavior. We also study the limit of large $q$ 's and find that in this case the exponent $\Omega$ obeys a simple power law rule.

Finally, our results can be used to draw conclusions on the 'dual black string system' obtained by appropriate double analytical continuations. This maps the bubble solution into a charged black string solution. Thus, exponential growing modes off the bubble solution turn out to correspond to static, harmonic 'deformations' of the uniform black strings. For these modes to exist, the strings length must be long enough to accommodate it, and we find that it must be an integer multiple of the critical length $L_{c}=2 \pi R_{0} / \Omega$. $L_{c}$ results exactly that expected from the Gregory Laflamme instability for the uncharged black strings 11]. For the charged case, however, the black string solutions we found are not the same as those presented in Ref. 12] as we here only consider $\mathbf{U}(\mathbf{1})$ gauge fields in the absence of a dilaton. Additionally, our results can be used to derive in a new way the exponential law for the critical mass presented by Sorkin [17] and to generalize it to the charged case. In particular, in the latter case we show how the addition of charge worsens the situation as far as stability of the string is concerned.

This work is organized as follows. In section ஹ we summarize the obtained results and discuss the implications for black string spacetimes. The rest of the article presents the details of our calculations. In section III we consider Kaluza-Klein theories coupled to a Maxwell field in spacetime dimensions $q+3 \geq 5$ with $\mathbf{U}(\mathbf{1}) \times \mathbf{S O}(\mathbf{q}+\mathbf{1})$ symmetry. We perform a dimensional reduction of the action, obtaining an effective action in two spacetimes dimension, and write 
down the equations of motions. In section IV we discuss the class of static solutions which represent the critical bubbles. The linear stability analysis about the bubble solutions follows in section $\nabla$ where we cast the perturbation equations into the form of a wave equation whose spatial operator is self-adjoint. We then show analytically that all bubble solutions are linearly unstable, and give analytic arguments for the existence of a unique non-degenerated eigenvalue $\lambda=-\Omega^{2} / R_{0}^{2}$ which is negative. Furthermore, we show that for fixed spacetime dimension, the corresponding frequency $\Omega$ is independent of the background solution. We provide analytical estimates for $\Omega$ and compute it by numerical means.

\section{CRITICAL BUBBLES AND BLACK STRINGS. RESULTS AND IMPLICATIONS}

\section{A. Critical bubbles: Summary of results}

In this article, we study the linear stability of the following static bubble solutions which are solution of the Einstein-Maxwell equations in $(q+3)$ spacetime dimensions:

$$
\begin{aligned}
d s^{2} & =V^{2 / q}\left[-d t^{2}+\frac{d R^{2}}{U}+R^{2} \hat{g}\right]+\frac{U}{V^{2}} d z^{2}, \\
A_{\mu} d x^{\mu} & =P \frac{1-U}{V} d z,
\end{aligned}
$$

where $P^{2}=\epsilon(1-\epsilon)(q+1) /\left(q^{2} \kappa\right), \kappa$ denoting a coupling constant, and where

$$
U=1-\left(R_{0} / R\right)^{q-1}, \quad V=1-\epsilon\left(R_{0} / R\right)^{q-1} .
$$

Here, $\hat{g}$ denotes the standard metric on the $q$-sphere $S^{q}$, and the coordinate $R$ lies in the interval $\left[R_{0}, \infty\right)$. The parameters $R_{0}>0$ (corresponding to the position of the bubble) and $\epsilon \in[0,1)$ are related to the ADM mass and to the asymptotic period $T$ of the extra dimension via $M_{A D M}=R_{0}^{q-1} T\left|S^{q}\right| / 16 \pi$ and $T=4 \pi R_{0}(1-\epsilon)^{1+1 / q} /(q-1)$.

We find spherically symmetric, in $z$ homogeneous, linear modes that depart like $\exp \left(\Omega t / R_{0}\right)$ from the static solution. For the uncharged case with $q=2$ this result can be drawn from the work [13] though here we concern ourselves to more generic cases. For each value of the spacetime dimension parameter $q$ we show that there is a unique such mode and that the corresponding dimensionless growth rate $\Omega$ does not depend on the parameter $\epsilon$ which is determining the strength of the gauge field. This universal behavior is precisely what is expected on physical grounds for a critical solution 30]. It explains the critical phenomena found in our numerical simulations [9, 10] where the universal parameter $\Gamma$ is related to $\Omega$ via $\Gamma=1 / \Omega$, i.e. $\Gamma$ is the inverse of the Lyapunov exponent of the single unstable mode. Table $\llbracket$ and figure 1 show the obtained numerical results for $\Omega$ as a function of $q$. Furthermore, our analysis reveals that that for $q$ large enough, $\Omega$ is approximately given by $\Omega \simeq q^{1 / 2}$. Figure 2 displays the percentile error $P E=100\left|\Omega-q^{1 / 2}\right| / q^{1 / 2}$.

\begin{tabular}{|l||c|c|c|c|c|c|c|c|c|c|c|c|c|c|}
\hline$q$ & 2 & 3 & 4 & 5 & 6 & 7 & 8 & 9 & 10 & 20 & 30 & 40 & 50 & 100 \\
\hline$\Omega$ & 0.876 & 1.27 & 1.58 & 1.85 & 2.09 & 2.30 & 2.50 & 2.69 & 2.87 & 4.26 & 5.30 & 6.17 & 6.93 & 9.90 \\
\hline
\end{tabular}

TABLE I: Numerical values for $\Omega$ for different values of $q$.

Summarizing, bubble spacetimes admit the critical solutions (12) for spacetime dimensions $q+3 \geq 5$ with corresponding critical exponent $\Omega$ which is independent of $\epsilon$. Furthermore, for $q$ large enough, $\Omega \simeq q^{1 / 2}$. Indeed, even at $q=7$ this approximation differs only by $\simeq 15 \%$ from the actual critical value.

\section{B. Implications for black strings}

Connections between possible black string instabilities and modes in a 'dual' space obtained by Wick rotations have been noted, and employed, in the past (see Refs. [13, 15, 16]). We here illustrate how one can make the connection between bubble and black string spacetimes via double-Wick rotations to draw conclusions for the latter case.

The bubble configurations (12) are related to a family of charged black string solutions via the double analytical continuation $t \mapsto i z, z \mapsto i t$ followed by the transformation $\epsilon \mapsto-\epsilon$ :

$$
d s^{2}=-\frac{U}{V^{2}} d t^{2}+V^{2 / q}\left(\frac{d R^{2}}{U}+d z^{2}+R^{2} \hat{g}\right)
$$




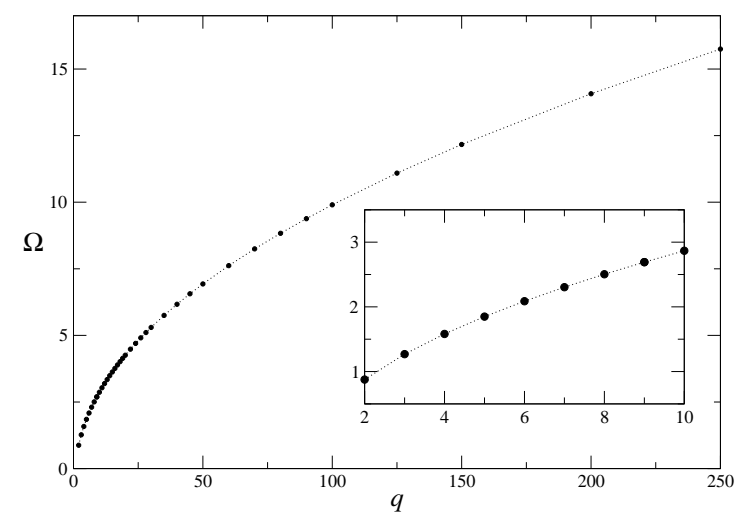

FIG. 1: The plot illustrates the obtained dependence of $\Omega$ on $q$ (dark circles, with a dotted joining line for guidance). The inset shows $\Omega$ versus $q$ for low values of $q$.

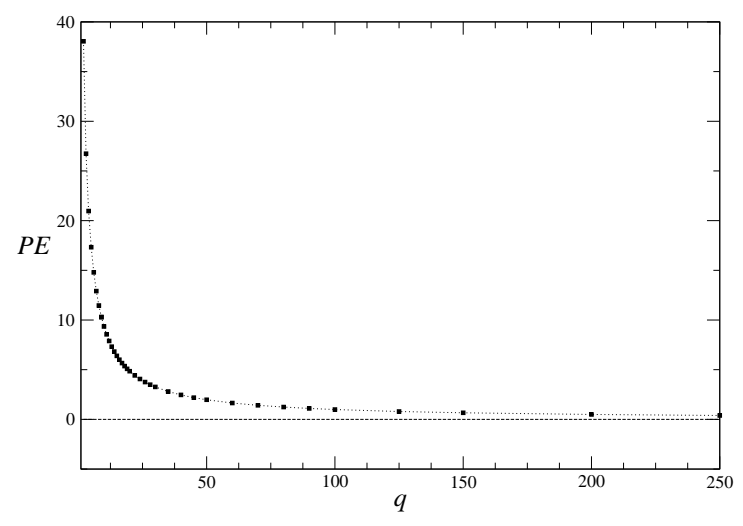

FIG. 2: Percentile error $P E$ of the actual value of $\Omega$ compared to the simple law $\sqrt{q}$

$$
A_{\mu} d x^{\mu}=-Q \frac{1-U}{V} d t
$$

where now $U=1-\left(R_{0} / R\right)^{q-1}, V=1+\epsilon\left(R_{0} / R\right)^{q-1}$, and $Q^{2}=\epsilon(1+\epsilon)(q+1) /\left(q^{2} \kappa\right)$. The parameters $R_{0}$ (which now corresponds to the location of the horizon) and $\epsilon$ satisfy $R_{0}>0, \epsilon \geq 0$ and are related to the mass and electric charge via

$$
\begin{aligned}
M & =\frac{R_{0}^{q-1} L\left|S^{q}\right|}{16 \pi}\left[q+2 \epsilon\left(q-\frac{1}{q}\right)\right], \\
Q_{e} & =\frac{(q-1) Q R_{0}^{q-1} L\left|S^{q}\right|}{4 \pi},
\end{aligned}
$$

where $L$ denotes the asymptotic period of the extra dimension. For $q=2$ these solutions agree with the solutions described by Eqs. (2.13) and (2.15) of Ref. [18].

Under the double analytical continuation, the unstable mode of the critical bubbles which is proportional to $e^{\Omega t / R_{0}}$ is mapped to a stationary mode of the corresponding black strings which is proportional to $e^{i \Omega z / R_{0}}$. This mode represents a static deformation of the black string, with harmonic dependency in $z$. That the existence of static modes are central in the understanding of instabilities has been discussed in [13, 16], we here exploit knowing these modes's wavelengths explicitly to draw conclusions of black string instabilities. Clearly, for this mode to exist, the asymptotic periodicity of the extra dimension must be an integer multiple of $L_{c}=2 \pi R_{0} / \Omega$. In the uncharged case, it turns out that $L_{c}$ is exactly equal to the critical length for the Gregory-Laflamme instability [11, 12]. (Compare the values of Table \with the values in Table 1 of Ref. 20].) For the charged case the critical solution does not correspond 
to the charged black string background solutions considered in 12 as ours only include the interaction with a $U(1)$ gauge field. However, as in [12] we expect that the presence of a static deformation is related to a threshold between stable and unstable solutions.

We are able to give the dependency for the critical length in terms of the parameter $\epsilon$ in an analytic way since we have shown that $\Omega$ is independent on $\epsilon$. The result is conveniently expressed in terms of the dimensionless mass defined in 17$]$

$$
\mu:=\frac{M_{c}}{L_{c}^{q}}=\frac{\left|S^{q}\right|}{16 \pi}\left(\frac{\Omega}{2 \pi}\right)^{q-1}\left[q+2 \epsilon\left(q-\frac{1}{q}\right)\right] .
$$

For large $q$ we can approximate $\Omega \simeq q^{1 / 2}$ and find

$$
\mu \simeq \frac{\sqrt{q}}{16}\left(\frac{e}{2 \pi}\right)^{q / 2}(1+2 \epsilon)
$$

which provides a different way of deriving the law presented in Ref. [17, 20], and generalizes it to the electrically charged case.

Note that in the charged case, the string becomes "more unstable" in the sense that the critical length at which instabilities arise is shorter that the charge-free case. This is to be contrasted with the case studied in [12, 19] where the opposite occurs. In that case, however, the interaction with a magnetic field was considered while in the present an electric field is included instead.

\section{KALUZA-KLEIN REDUCTION AND EFFECTIVE ACTION}

We consider a $q+3$ dimensional spacetime $(M, g)$ with $\mathbf{U}(\mathbf{1}) \times \mathbf{S O}(\mathbf{q}+\mathbf{1})$ symmetry. We assume that the Killing field $\partial_{z}$ which is the generator of the action of the $\mathbf{U}(\mathbf{1})$ group on $M$ is hypersurface orthogonal. The manifold has the structure $M=\tilde{M} \times S^{q} \times S^{1}$ with metric

$$
d s^{2}=e^{-2 \phi / q}\left[\tilde{g}_{a b} d x^{a} d x^{b}+r^{2} \hat{g}\right]+e^{2 \phi} d z^{2}
$$

where $\phi, r$ and $\tilde{g}_{a b} d x^{a} d x^{b}$ are, respectively, a function, a positive function, and a pseudo-Riemannian metric on the two-dimensional manifold $\tilde{M}$. $\hat{g}$ denotes the standard metric on the $q$-dimensional sphere $S^{q}$. In this article, we assume that $q \geq 2$. We also consider a $\mathbf{U}(\mathbf{1})$ gauge potential of the form

$$
A_{\mu} d x^{\mu}=\gamma d z
$$

with $\gamma$ a function on $\tilde{M}$.

The dynamics is described by the $q+3$ dimensional Einstein-Hilbert-Maxwell action

$$
S=-\frac{1}{16 \pi} \int[R i c-q \kappa g(d A, d A)] \sqrt{-g} d^{q+3} x
$$

where Ric denotes the Ricci scalar belonging to the metric (8) and $\kappa$ is a coupling constant. When integrating over $z$, one obtains a reduced action describing $q+2$ dimensional gravity coupled to the dilaton field $\phi$ and to the $\mathbf{U}(\mathbf{1})$ gauge potential $\gamma d z$. Using the $S O(q+1)$ symmetry one can further reduce the action [31] and, after some manipulations, obtains

$$
S_{\text {eff }}=-\int\left[2 \frac{\tilde{g}(d m, d r)}{N}-\frac{q+1}{q^{2}} r^{q} \tilde{g}(d \phi, d \phi)-\kappa r^{q} e^{-2 \phi} \tilde{g}(d \gamma, d \gamma)\right] \sqrt{-\tilde{g}} d x^{0} \wedge d x^{1},
$$

where the functions $N$ and $m$ are defined by $N=\tilde{g}(d r, d r), N=1-2 m r^{1-q}$, and where $\left(x^{0}, x^{1}\right)$ are local coordinates on $\tilde{M}$. We stress that up to this point all the fields are defined in an invariant geometrical way: $e^{2 \phi}$ is the norm of the Killing field $\partial_{z}, r$ is defined via the area radius, $e^{-\phi / q} r$, of the $q$-spheres, and $N$ is the norm of $d r$.

In order to write down the field equations, we choose the coordinates $\left(x^{0}, x^{1}\right)$ such that $x^{1}=r$, set $t=x^{0}$ and parametrize the two-metric according to

$$
\tilde{g}_{a b} d x^{a} d x^{b}=-\sigma^{2} N(d t+a d r)^{2}+\frac{d r^{2}}{N}
$$


where $\sigma=\sqrt{-\tilde{g}}$ and $a=-\tilde{g}(d t, d r) / N$. The action has the form

$$
S_{e f f}=-2 \int\left[\mathcal{L}_{G}+\mathcal{L}_{M}\right] d t \wedge d r
$$

with

$$
\begin{aligned}
\mathcal{L}_{G} & =\sigma\left(m^{\prime}-a \dot{m}\right) \\
\mathcal{L}_{M} & =\frac{\sigma r^{q}}{2}\left[\frac{\dot{\psi}^{2}}{\sigma^{2} N}-N\left(\psi^{\prime}-a \dot{\psi}\right)^{2}\right]+\frac{\kappa \sigma r^{q} e^{-\alpha \psi}}{2}\left[\frac{\dot{\gamma}^{2}}{\sigma^{2} N}-N\left(\gamma^{\prime}-a \dot{\gamma}\right)^{2}\right]
\end{aligned}
$$

where we have defined $\psi=\sqrt{(q+1) / q^{2}} \phi, \alpha=2 q / \sqrt{q+1}$ for convenience, and where a dot and a prime denote differentiation with respect to $t$ and $r$, respectively.

Of course, we can always choose the coordinate $t$ such that $a=0$. However, we shall set $a=0$ only after varying the action since otherwise an equation is lost. The field equations obtained from varying (13) with respect to $\sigma, m$, $a, \psi$ and $\gamma$ are, after setting $a=0$,

$$
\begin{aligned}
2 m^{\prime} & =r^{q}\left[\frac{\dot{\psi}^{2}}{\sigma^{2} N}+N \psi^{\prime 2}\right]+\kappa r^{q} e^{-\alpha \psi}\left[\frac{\dot{\gamma}^{2}}{\sigma^{2} N}+N \gamma^{\prime 2}\right], \\
N \frac{\sigma^{\prime}}{\sigma} & =r\left[\frac{\dot{\psi}^{2}}{\sigma^{2} N}+N \psi^{\prime 2}\right]+\kappa r e^{-\alpha \psi}\left[\frac{\dot{\gamma}^{2}}{\sigma^{2} N}+N \gamma^{\prime 2}\right], \\
\dot{m} & =r^{q} N \dot{\psi} \psi^{\prime}+\kappa r^{q} N e^{-\alpha \psi} \dot{\gamma} \gamma^{\prime},
\end{aligned}
$$

and

$$
\begin{aligned}
& r^{q} \partial_{t}\left(\frac{\dot{\psi}}{\sigma N}\right)-\left(r^{q} \sigma N \psi^{\prime}\right)^{\prime}=-\frac{\alpha \kappa}{2} \sigma r^{q} e^{-\alpha \psi}\left[\frac{\dot{\gamma}^{2}}{\sigma^{2} N}-N \gamma^{\prime 2}\right] \\
& r^{q} \partial_{t}\left(e^{-\alpha \psi} \frac{\dot{\gamma}}{\sigma N}\right)-\left(r^{q} \sigma N e^{-\alpha \psi} \gamma^{\prime}\right)^{\prime}=0 .
\end{aligned}
$$

Combining Eqs. (16) and (17) we also find

$$
\frac{1}{\sigma}(\sigma N)^{\prime}=(q-1) \frac{2 m}{r^{q}}
$$

In the next section, we discuss static solutions to these equations, where $\dot{m}=\dot{\psi}=\dot{\gamma}=0$.

\section{STATIC SOLUTIONS}

When $\gamma=\psi=0$ we obtain $m=$ const, so the only solutions to the field equations consists of the one-parameter family of $q+2$ dimensional Schwarzschild spacetimes cross $S^{1}$. For static configurations in general, we notice that Eqs. (19), (20) posses the first integrals

$$
\begin{aligned}
& r^{q} \sigma N e^{-\alpha \psi} \gamma^{\prime}=\tilde{P}, \\
& r^{q} \sigma N \psi^{\prime}+\frac{1}{2} \alpha \kappa \tilde{P} \gamma=\kappa \tilde{Q},
\end{aligned}
$$

with two constants $\tilde{P}, \tilde{Q}$. In fact, the static equations can be integrated completely for bubble configurations obtaining the following two-parameter family of solutions,

$$
\begin{aligned}
d s^{2} & =\frac{V^{2 / q}}{U^{1 / q}}\left[-U^{1 / q} d t^{2}+U^{1 / q-1} d R^{2}+R^{2} U^{1 / q} \hat{g}\right]+\frac{U}{V^{2}} d z^{2} \\
A_{\mu} d x^{\mu} & =\frac{P}{\epsilon V} d z
\end{aligned}
$$


where $P^{2}=\epsilon(1-\epsilon)(q+1) /\left(q^{2} \kappa\right)$, and where

$$
U=1-\left(R_{0} / R\right)^{q-1}, \quad V=1-\epsilon\left(R_{0} / R\right)^{q-1} .
$$

The coordinate $R$ lies in the open interval $\left[R_{0}, \infty\right.$ ). The parameters $R_{0}>0$ (corresponding to the position of the bubble) and $\epsilon \in[0,1)$ are related to the ADM mass and to the asymptotic period $T$ of the extra dimension, see section III In order to determine the latter, we replace the coordinate $R$ by the coordinate $\hat{\rho}$ defined by $U=\hat{\rho}^{2}$ and rewrite

$$
\frac{V^{2 / q}}{U} d R^{2}+\frac{U}{V^{2}} d z^{2}=\frac{1}{V^{2}}\left(\frac{4 R_{0}^{2}}{(q-1)^{2}}\left(1-\epsilon+\epsilon \hat{\rho}^{2}\right)^{\frac{2(q+1)}{q}}\left(1-\hat{\rho}^{2}\right)^{-\frac{2 q}{q-1}} d \hat{\rho}^{2}+\hat{\rho}^{2} d z^{2}\right) .
$$

Therefore, in order to avoid a conical singularity at $\hat{\rho}=0$, we need $T=4 \pi R_{0}(1-\epsilon)^{1+1 / q} /(q-1)$. The integration constants $\tilde{P}, \tilde{Q}$ are related to $R_{0}$ and $P$ via

$$
\tilde{P}=-(q-1) R_{0}^{q-1} P, \quad \tilde{Q}=-\frac{(q-1) R_{0}^{q-1}}{\alpha \kappa} .
$$

In the next section we consider time-dependent bubble configurations that are infinitesimally close to the static solutions. That is, we consider metrics of the form

$$
d s^{2}=\left(\frac{V^{2}}{U} e^{-2 E}\right)^{1 / q}\left[-U^{1 / q} e^{2 A} d t^{2}+U^{1 / q-1} e^{2 B} d R^{2}+R^{2} U^{1 / q} e^{2 C} \hat{g}\right]+\frac{U}{V^{2}} e^{2 E} d z^{2},
$$

where $A, B, C, E$ are "small" functions of $t$ and $R$, which vanish as $R \rightarrow \infty$ and such that $B\left(t, R_{0}\right)=(1+1 / q) E\left(t, R_{0}\right)$. The former conditions are necessary for asymptotic flatness while the latter ensures that the period of the extra dimension $T$ does not change. In order to determine the asymptotic behavior in the amplitudes $\sigma, N, \psi$ and $\gamma$ we notice that

$$
\begin{aligned}
e^{\alpha \psi} & =\frac{U}{V^{2}} e^{2 E}, \\
r & =R U^{1 / 2 q} e^{C}, \\
N & =\tilde{g}(d r, d r)=U^{1-1 / q} e^{-2 B}\left[\frac{\partial r}{\partial R}\right]^{2}, \\
\sigma^{2} N & =U^{1 / q} e^{2 A}, \\
\gamma & =\frac{P}{\epsilon V}+\delta \gamma .
\end{aligned}
$$

Clearly, this implies that $\psi \rightarrow 0, \sigma, N \rightarrow 1$ as $R \rightarrow \infty$. In order to analyze the behavior near the bubble, we introduce the dimensionless quantity $x=\left(R-R_{0}\right) / R_{0}$. The background quantities (the ones obtained by setting $A=B=C=E=0$ ) have the expansion

$$
\begin{aligned}
e^{\alpha \psi} & =\frac{(q-1) x}{(1-\epsilon)^{2}}+O\left(x^{2}\right), \\
r^{2 q} & =R_{0}^{2 q}(q-1) x+O\left(x^{2}\right), \\
\sigma & =2 q(q-1)^{-\frac{q-1}{2 q}} x^{\frac{q+1}{2 q}}[1+O(x)], \\
N & =\frac{(q-1)}{4 q^{2}} \frac{1}{x}+O(1) .
\end{aligned}
$$

Notice that $N$ diverges as $R \rightarrow R_{0}$, but $\sigma^{2} N$ goes to zero. Linearizing about the background, we find that $\delta C=0$ (since we do not vary $r$ ), and $\delta \psi=2 \alpha^{-1} \delta E, \delta N=-2 N \delta B$. As a consequence of this,

$$
\left.\sigma \delta m\right|_{R=R_{0}}=\frac{q-1}{2 q} R_{0}^{q-1} \delta B\left(t, R_{0}\right)=\frac{q^{2}-1}{2 q^{2}} R_{0}^{q-1} \delta E\left(t, R_{0}\right) .
$$

Here and in the following, $\delta(\ldots)$ denotes the linearization of the quantity $(\ldots)$ about a static background. 


\section{LINEAR PERTURBATIONS}

Since when $\gamma=\psi=0$ all solutions are static, and in view of the wave-like character of the equations (19), (20) for $\psi$ and $\gamma$, we expect that the true dynamical degrees of freedom are contained in $\gamma$ and $\psi$. It turns out that this expectation is indeed true at least at the perturbative level: Our effective action Eq. (13) satisfies the form of Ref. 21] where it was shown that for linear perturbations around a static background the "gravitational" degrees of freedom can be completely eliminated and a pulsation equation for the perturbed matter fields alone can be obtained. In order to see this explicitly, we first linearize Eqs. (18) and (16), obtaining

$$
\begin{aligned}
\delta \dot{m} & =r^{q} N\left(\psi^{\prime} \delta \dot{\psi}+\kappa e^{-\alpha \psi} \gamma^{\prime} \delta \dot{\gamma}\right), \\
\delta m^{\prime} & =r^{q} N\left(\psi^{\prime} \delta \psi^{\prime}+\kappa e^{-\alpha \psi} \gamma^{\prime} \delta \gamma^{\prime}\right)+\frac{r^{q} \delta N}{2}\left(\psi^{\prime 2}+\kappa e^{-\alpha \psi} \gamma^{\prime 2}\right)-\frac{\alpha \kappa}{2} r^{q} N e^{-\alpha \psi} \gamma^{\prime 2} \delta \psi .
\end{aligned}
$$

Using $r^{q} \delta N=-2 r \delta m$ and the background equation $\sigma^{\prime} / \sigma=r \psi^{\prime 2}+\kappa r e^{-\alpha \psi} \gamma^{\prime 2}$ we can rewrite the second equation as

$$
[\sigma \delta m]^{\prime}=r^{q} \sigma N\left(\psi^{\prime} \delta \psi^{\prime}+\kappa e^{-\alpha \psi} \gamma^{\prime} \delta \gamma^{\prime}\right)-\frac{\alpha \kappa}{2} r^{q} \sigma N e^{-\alpha \psi} \gamma^{\prime 2} \delta \psi
$$

Finally, using the static version of Eqs. (19), (20), we find

$$
[\sigma \delta m]^{\prime}=\left[r^{q} \sigma N\left(\psi^{\prime} \delta \psi+\kappa e^{-\alpha \psi} \gamma^{\prime} \delta \gamma\right)\right]^{\prime}
$$

Equations (36) and (39) imply that

$$
\sigma \delta m=r^{q} \sigma N\left(\psi^{\prime} \delta \psi+\kappa e^{-\alpha \psi} \gamma^{\prime} \delta \gamma\right)+\text { const. }
$$

which, using Eqs. (22) and (23), can also be written as

$$
\sigma \delta m=\kappa\left(\tilde{Q}-\frac{1}{2} \alpha \tilde{P} \gamma\right) \delta \psi+\tilde{P} \delta \gamma+\text { const }
$$

In order to determine the constant, we evaluate this equation at $R=R_{0}$. Taking into account Eq. (35) we obtain $0=\tilde{P} \delta \gamma\left(t, R_{0}\right)+$ const. Since the addition of a constant to $\delta \gamma$ is a gauge transformation, we set the constant to zero in the following, which is equivalent to requiring $\delta \gamma\left(t, R_{0}\right)=0$. Using Eq. (41) and the linearization of Eq. (21), we also find

$$
\delta\left[\frac{(\sigma N)^{\prime}}{\sigma N}\right]=\frac{2(q-1)}{N}\left(\psi^{\prime} \delta \psi+\kappa e^{-\alpha \psi} \gamma^{\prime} \delta \gamma\right) .
$$

Eq. (42) allow to reexpress all gravitational perturbations in terms of matter perturbations in the linearized versions of Eqs. (19) and (20). Therefore, as anticipated, the linearized "gravitational" degrees of freedom can be entirely expressed in terms of the linearized "matter" fields, and one obtains the following pulsation equation

$$
\mathbf{P} \ddot{v}-\left(\mathbf{N}^{T}\right)^{-1} \sigma N \partial_{r}\left[\sigma N \mathbf{N}^{T} \mathbf{P} \mathbf{N} \partial_{r}\left(\mathbf{N}^{-1} v\right)\right]+\mathbf{S} v=0
$$

with

$$
\begin{aligned}
& \mathbf{P}=r^{q}\left(\begin{array}{cc}
1 & 0 \\
0 & e^{-\alpha \psi}
\end{array}\right), \\
& \mathbf{S}=-\frac{\alpha^{2} \kappa \tilde{P}^{2} e^{\alpha \psi}}{2 r^{q}}\left(\begin{array}{ll}
1 & 0 \\
0 & 0
\end{array}\right)-\frac{2(q-1)}{r^{q} N}\left(\begin{array}{c}
\kappa \tilde{Q}-\frac{1}{2} \alpha \kappa \tilde{P} \gamma \\
\sqrt{\kappa} \tilde{P}
\end{array}\right)\left(\kappa \tilde{Q}-\frac{1}{2} \alpha \kappa \tilde{P} \gamma, \sqrt{\kappa} \tilde{P}\right), \\
& \mathbf{N}=\left(\begin{array}{cc}
1 & 0 \\
\alpha \sqrt{\kappa} \gamma & 1
\end{array}\right),
\end{aligned}
$$

and where $v=(\delta \psi, \sqrt{\kappa} \delta \gamma)^{T}$. The spatial part of the operator acting on $v$ in Eq. (43) is a formally self-adjoint elliptic operator. In the following, we start with a qualitative analysis of this operator, introducing a Hilbert space on which it is defined, and giving estimates for the lower limit of its spectrum. Then, we prove the existence of a unique bound state, i.e. an eigenfunction belonging to a negative eigenvalue. In this sense, each bubble configuration possesses precisely one unstable mode. The timescale associated to this mode explains the critical exponent in the critical dynamical behavior that has been announced in Ref. 99] and that will be discussed in detail in Ref. [10]. 


\section{A. Qualitative analysis of the pulsation operator}

Here we give a first analysis of the pulsation operator which is defined by the spatial part of the operator acting on $v$ in Eq. (43). In order to do so, we first rewrite the pulsation equations in terms of the dimensionless quantities $\bar{R}=R / R_{0}, \bar{t}=t / R_{0}, q$ and $\epsilon$. Using the relations in section IV we find

$$
\left(\frac{\partial^{2}}{\partial \bar{t}^{2}}+\mathcal{A}\right) v=0
$$

where the pulsation operator is defined by

$$
\mathcal{A}=-\overline{\mathbf{P}}^{-1}\left(\mathbf{N}^{T}\right)^{-1} \frac{\partial}{\partial \bar{R}}\left[U \mathbf{N}^{T} \overline{\mathbf{P}} \mathbf{N} \frac{\partial}{\partial \bar{R}}\left(\mathbf{N}^{-1} \cdot\right)\right]+\overline{\mathbf{P}}^{-1} \overline{\mathbf{S}}
$$

where

$$
\begin{aligned}
& \overline{\mathbf{P}}=\bar{R}^{q}\left(\begin{array}{cc}
1 & 0 \\
0 & \frac{V^{2}}{U}
\end{array}\right) \\
& \overline{\mathbf{S}}=-\frac{2(q-1)^{2}}{\bar{R}^{q}} \frac{\epsilon(1-\epsilon)}{V^{2}}\left(\begin{array}{ll}
1 & 0 \\
0 & 0
\end{array}\right)-\frac{2\left(q^{2}-1\right)}{\bar{R}^{q}\left[1+\frac{q+1}{q-1} U\right]^{2}}\left(\begin{array}{c}
1-2(1-\epsilon) V^{-1} \\
2 \sqrt{\epsilon(1-\epsilon)}
\end{array}\right)\left(1-2(1-\epsilon) V^{-1}, 2 \sqrt{\epsilon(1-\epsilon)}\right) \\
& \mathbf{N}=\left(\begin{array}{cc}
1 & 0 \\
2 \sqrt{\frac{1-\epsilon}{\epsilon}} V^{-1} & 1
\end{array}\right)
\end{aligned}
$$

and where

$$
U=1-\bar{R}^{-(q-1)}, \quad V=1-\epsilon \bar{R}^{-(q-1)}
$$

In order to discuss the pulsation equation, we define an appropriate Hilbert space in which the operator $\mathcal{A}$ (defined on a suitable domain) is self-adjoint. The solutions to Eq. (44) can then be constructed using spectral theory, and the long-time behavior of the solutions is determined by the spectrum of $\mathcal{A}$. We define the Hilbert space

$$
\mathcal{H}=L^{2}\left((1, \infty), \mathbb{R}^{2} ; \overline{\mathbf{P}} d \bar{R}\right)
$$

of all functions $v:(1, \infty) \rightarrow \mathbb{R}^{2}$ which are square integrable with respect to the measure $\overline{\mathbf{P}} d \bar{R}$. The corresponding scalar product is given by

$$
(u, v)=\int_{1}^{\infty} u^{T} \overline{\mathbf{P}} v d \bar{R}
$$

Let $u, v$ be smooth functions on $(1, \infty)$. Using integration by parts we obtain

$$
(u, \mathcal{A} v)=\int_{1}^{\infty}\left[\frac{\partial}{\partial \bar{R}}\left(\mathbf{N}^{-1} u\right)^{T} \cdot U \mathbf{N}^{T} \overline{\mathbf{P}} \mathbf{N} \frac{\partial}{\partial \bar{R}}\left(\mathbf{N}^{-1} v\right)+u^{T} \overline{\mathbf{S}} v\right] d \bar{R}-\left.u^{T} U \overline{\mathbf{P}} \mathbf{N} \frac{\partial}{\partial \bar{R}}\left(\mathbf{N}^{-1} v\right)\right|_{\bar{R}=1} ^{\infty} .
$$

Defining the domain $D(\mathcal{A})$ of $\mathcal{A}$ to be the set of all smooth functions $u:(1, \infty) \rightarrow \mathbb{R}^{2}$ such that all derivatives are uniformly bounded on $(1, \infty)$ and such that $u_{2}(\bar{R}=1)=0$ and $u$ and its derivatives decay faster than $\bar{R}^{-q / 2-1 / 2}$ as $\bar{R} \rightarrow \infty$, we see that the boundary term vanishes for $u, v \in D(\mathcal{A})$, and so

$$
(u, \mathcal{A} v)=(\mathcal{A} u, v)
$$

for all $u, v \in D(\mathcal{A})$. Therefore, $\mathcal{A}: D(\mathcal{A}) \subset \mathcal{H} \rightarrow \mathcal{H}$ is a densely defined, symmetric operator. Since $\mathcal{A}$ is real, in the sense that it commutes with complex conjugation, $\mathcal{A}$ can be extended to a self-adjoint operator; that is, $\mathcal{A}$ can be defined on a larger domain $D(\hat{\mathcal{A}}) \supset D(\mathcal{A})$ to yield a self-adjoint operator $\hat{\mathcal{A}}: D(\hat{\mathcal{A}}) \subset \mathcal{H} \rightarrow \mathcal{H}$. In general, there might exist different such self-adjoint extensions. If $\mathcal{A}$ is bounded from below, one can single out an extension by requiring that it possesses the same lower bound as $\mathcal{A}$. In the following, we show that $\mathcal{A}$ is bounded from below, and take as extension $\hat{\mathcal{A}}$ this unique self-adjoint extension. We refer the reader to Ref. [22] for the theory of self-adjoint extensions of densely defined symmetric operators. 
In order to show that $\mathcal{A}$ is bounded from below, and in order to give an estimate for this lower bound, we estimate the quantity

$$
G \equiv \inf _{\substack{v \in D(\mathcal{A}) \\ v \neq 0}} \frac{(v, \mathcal{A} v)}{(v, v)} .
$$

We first show that $G>-\infty$ which implies that $\mathcal{A}$ is bounded from below and so there is a unique self-adjoint extension $\hat{\mathcal{A}}$ which has the same lower bound $G$. We will also show that $G$ is negative which means that the background solutions are unstable. Furthermore, we will show that $\hat{\mathcal{A}}$ possesses precisely one eigenfunction with negative eigenvalue. In this subsection we provide estimates for this eigenvalue; its existence and uniqueness and the numerical computation of its actual value are given in the next subsection.

Our analysis of the pulsation operator $\mathcal{A}$ is greatly simplified by performing the transformation $v \mapsto \mathbf{O} v$ where the matrix $\mathbf{O}$ is given by

$$
\mathbf{O}=\left(\begin{array}{cc}
1-2(1-\epsilon) V^{-1} & -2 \sqrt{\epsilon(1-\epsilon)} \\
2 \sqrt{\epsilon(1-\epsilon)} U V^{-2} & 1-2(1-\epsilon) V^{-1}
\end{array}\right) .
$$

Notice that $\mathbf{O}$ defines an orthogonal transformation with respect to the scalar product defined in (46) since $\mathbf{O}^{T} \overline{\mathbf{P}} \mathbf{O}=$ $\overline{\mathbf{P}}$. Furthermore, $\mathbf{O}$ leaves the domain $D(\mathcal{A})$ of $\mathcal{A}$ invariant. Remarkably, the transformation $\mathbf{O}$ brings the pulsation operator into diagonal form:

$$
\mathbf{O}^{\dagger} \mathcal{A} \mathbf{O}=\overline{\mathbf{P}}^{-1}\left(\begin{array}{cc}
\mathcal{B}_{1} & 0 \\
0 & \mathcal{B}_{2}
\end{array}\right)
$$

where $\mathbf{O}^{\dagger}=\overline{\mathbf{P}}^{-1} \mathbf{O}^{T} \overline{\mathbf{P}}=\mathbf{O}^{-1}$ and

$$
\begin{aligned}
& \mathcal{B}_{1}=-\frac{\partial}{\partial \bar{R}} U \bar{R}^{q} \frac{\partial}{\partial \bar{R}}-\frac{2\left(q^{2}-1\right)}{\bar{R}^{q}\left[1+\frac{q+1}{q-1} U\right]^{2}}, \\
& \mathcal{B}_{2}=-\frac{\partial}{\partial \bar{R}} V^{2} \bar{R}^{q} \frac{\partial}{\partial \bar{R}} .
\end{aligned}
$$

Therefore, it is sufficient to analyze the two decoupled eigenvalue problems $\mathcal{B}_{1} u_{1}=\lambda \bar{R}^{q} u_{1}, \mathcal{B}_{2} u_{2}=\lambda \bar{R}^{q} V^{2} U^{-1} u_{2}$, $\left(u_{1}, u_{2}\right)^{T} \in D(\mathcal{A})$, with $\lambda \in \mathbb{R}$ an eigenvalue. Since $\mathcal{B}_{2}$ is a positive operator, only the operator $\mathcal{B}_{1}$ can lead to instabilities. Notice that $\mathcal{B}_{1}$ does not depend on $\epsilon$. Therefore, the bound states of the pulsation operator (if they exist) are independent of $\epsilon$. In the following, we derive lower and upper bounds for the quantity

$$
G_{1} \equiv \inf _{\substack{u \in D(\mathcal{A}) \\ u \neq 0}} \frac{\int_{1}^{\infty} u_{1} \mathcal{B}_{1} u_{1} d \bar{R}}{\int_{1}^{\infty} \bar{R}^{q} u_{1}^{2} d \bar{R}} .
$$

Since the transformation $\mathbf{O}$ is orthogonal, $\mathcal{B}_{2}$ is positive, and since we will show that $G_{1}$ is negative, it follows that $G=G_{1}$, so $G_{1}$ gives the lower limit of the spectrum of the pulsation operator.

Using integration by parts, we find, for $u \in D(\mathcal{A})$ :

$$
\begin{aligned}
\int_{1}^{\infty} u_{1} \mathcal{B}_{1} u_{1} d \bar{R} & =\int_{1}^{\infty}\left[U \bar{R}^{q}\left(\frac{\partial u_{1}}{\partial \bar{R}}\right)^{2}-\frac{2\left(q^{2}-1\right) u_{1}^{2}}{\bar{R}^{q}\left[1+\frac{q+1}{q-1} U\right]^{2}}\right] d \bar{R} \\
& \geq-2\left(q^{2}-1\right) \int_{1}^{\infty} \bar{R}^{q} u_{1}^{2} d \bar{R},
\end{aligned}
$$

which shows that $G_{1} \geq-2\left(q^{2}-1\right)$. Next, we derive upper bounds for $G_{1}$. In order to do so, we introduce the quantities

$$
\begin{aligned}
& Z=(q-1) \int_{1}^{\infty} \bar{R}^{q} u_{1}^{2} d \bar{R}=\int_{0}^{1}(1-U)^{-\frac{2 q}{q-1}} u_{1}^{2} d U \\
& E=\frac{1}{q-1} \int_{1}^{\infty} u_{1} \mathcal{B}_{1} u_{1} d \bar{R}=\int_{0}^{1}\left[U\left(\frac{\partial u_{1}}{\partial U}\right)^{2}-2 \frac{q+1}{q-1} \frac{u_{1}^{2}}{\left[1+\frac{q+1}{q-1} U\right]^{2}}\right] d U,
\end{aligned}
$$


and minimize the functional $u_{1} \mapsto E / Z$ over suitable test functions. We first study the limit $q \rightarrow \infty$. In this limit, $E$ becomes positive. Indeed, using integration by parts and requiring $Z<\infty$, we can rewrite

$$
E=\int_{0}^{1} U\left[\frac{1-U}{1+U} \frac{\partial}{\partial U}\left(\frac{1+U}{1-U} u_{1}\right)\right]^{2} d U .
$$

Therefore, in the limit $q \rightarrow \infty, E \geq 0$ and $E=0$ if and only if $u_{1}=$ const $\cdot(1-U) /(1+U)$. For $3<q<\infty$, this motivates the following test functions:

$$
u_{1}=\frac{1-U}{1+\frac{q+1}{q-1} U}
$$

This yields

$$
Z=\int_{0}^{1} \frac{(1-U)^{-\frac{2}{q-1}}}{\left[1+\frac{q+1}{q-1} U\right]^{2}} d U \leq \int_{0}^{1}(1-U)^{-\frac{2}{q-1}} d U=\frac{q-1}{q-3}
$$

and $E=-1 /(2 q)$. Therefore,

$$
G_{1} \leq(q-1)^{2} \frac{E}{Z} \leq-\frac{(q-1)(q-3)}{2 q}<0 .
$$

Asymptotically as $q \rightarrow \infty$, we have $Z \simeq 1 / 2$, and the test function $u_{1}$ defined in Eq. [55] becomes optimal in this limit, so we expect that for $q>>1$,

$$
G_{1} \simeq-q .
$$

The numerical results in the next subsection will confirm this behavior.

For $q=2$ or $q=3$, the decay of $u_{1}$ defined in Eq. (55) as $U$ approaches 1 is not rapid enough for $Z$ to be finite. For this reason, we define $u_{1}=(1-U)^{m} /[1+(q+1) U /(q-1)]$ with $m=3$ for $q=2$ and $m=2$ for $q=3$. These test functions yield the estimates

$$
\begin{aligned}
& G_{1} \leq-\frac{1}{9} \frac{381-512 \log (2)}{15-16 \log (2)} \approx-0.74 \quad \text { for } q=2, \\
& G_{1} \leq-2 \frac{4-3 \log (3)}{2-\log (3)} \approx-1.56 \quad \text { for } q=3 .
\end{aligned}
$$

As we will see in the next subsection, these bounds are very close to the actual eigenvalue.

To summarize, we have shown that the pulsation operator defines a self-adjoint operator whose spectrum is contained in the interval $\left[-2\left(q^{2}-1\right), \infty\right)$. We have also shown that the lower limit of the spectrum is negative, and is independent of $\epsilon$. In particular, this proves that for all $0 \leq \epsilon<1, q \geq 2$, the static bubble solutions are unstable. In the next subsection we show the existence of a unique non-degenerated negative eigenvalue for each $q \geq 2$.

\section{B. Quantitative analysis - eigenvalues of $\mathcal{A}$}

We consider the eigenvalue problem

$$
-\left(U \bar{R}^{q} w^{\prime}\right)^{\prime}-\frac{2\left(q^{2}-1\right)}{\bar{R}^{q}\left[1+\frac{q+1}{q-1} U\right]^{2}} w=\lambda \bar{R}^{q} w,
$$

where here and in the following, a prime denotes differentiation with respect to $\bar{R}$, and $w$ is a smooth function on $(1, \infty)$ such that all its derivatives are bounded near $\bar{R}=1$ and decay faster than $\bar{R}^{-q / 2-1 / 2}$ near $\bar{R}=\infty$. We show that there exists precisely one negative eigenvalue $\lambda$ to this problem and compute it by numerical means. The following analysis is standard and follows the theory of singular Sturm-Liouville problems [23].

We first show that there exists local solutions with the required behavior near $\bar{R}=1$ and $\bar{R}=\infty$. At the location of the bubble $(\bar{R}=1)$, we have a regular singular point while at $\bar{R}=\infty$ we have an essential singularity. In order to 
analyze the behavior near $\bar{R}=1$, it is convenient to replace the coordinate $\bar{R}$ by $U=1-\bar{R}^{-(q-1)}$. We then obtain the system

$$
U \frac{\partial \Phi}{\partial U}=A(U) \Phi, \quad \Phi=\left(\begin{array}{c}
w \\
U \frac{\partial w}{\partial U}
\end{array}\right)
$$

where

$$
A(U)=\left(\begin{array}{cc}
0 & 1 \\
-2 \frac{q+1}{q-1} \frac{U}{\left[1+\frac{q+1}{q-1} U\right]^{2}}-\frac{\lambda U}{(q-1)^{2}}(1-U)^{-\frac{2 q}{q-1}} & 0
\end{array}\right) .
$$

For $0 \leq U<1, A(U)=\sum_{k \geq 0} A_{k} U^{k}$ is analytic in $U$ with

$$
A_{0}=\left(\begin{array}{ll}
0 & 1 \\
0 & 0
\end{array}\right), \quad A_{1}=\left(\begin{array}{cc}
0 & 0 \\
-2 \frac{q+1}{q-1}-\frac{\lambda}{(q-1)^{2}} & 0
\end{array}\right), \quad A_{2}=\ldots
$$

Therefore, to leading order in $U$, Eq. 61] reads

$$
\frac{\partial \Phi}{\partial(\log U)}=A_{0} \Phi
$$

with solution $\Phi_{0}=\left(1+A_{0} \log U\right) e_{0}$, where $e_{0}$ is a constant vector. Since we require $\Phi$ to be bounded near $U=0$ we have to choose $e_{0}=(1,0)$ in order to avoid the logarithmic term. According to the general theory of regular singular points [24], this gives rise to a one-parameter family of local solutions near $U=0$ of the form $\Phi=\sum_{k \geq 0} e_{k} U^{k}$. Plugging this form into Eq. (61) yields the recursion relation

$$
e_{k}=\left(k \mathbf{I}-A_{0}\right)^{-1} \sum_{l=1}^{k} A_{l} e_{k-l}, \quad k=1,2,3, \ldots,
$$

where I denotes the identity matrix. The series converges for $U>0$ small enough 24].

In order to analyze the behavior near $\bar{R}=\infty$, we first introduce the coordinate $\rho$ defined by

$$
\rho(\bar{R})=\int_{1}^{\bar{R}} \frac{d R}{\sqrt{U(R)}}, \quad \bar{R} \geq 1
$$

which runs from zero to infinity. Notice that $\rho / \bar{R} \rightarrow 1$ as $\bar{R} \rightarrow \infty$. Therefore, there is a smooth function $h(\rho)$ such that $h(\rho) \rightarrow 1$ as $\rho \rightarrow \infty$ and such that $\rho=h(\rho) \bar{R}$. In terms of this new coordinate, we can rewrite the eigenvalue problem (60) as

$$
\frac{\partial^{2}}{\partial \rho^{2}} X+(\lambda+B(\rho)) X=0, \quad B(\rho)=-\frac{1}{T} \frac{\partial^{2} T}{\partial \rho^{2}}+\frac{2\left(q^{2}-1\right)}{\bar{R}^{2 q}\left[1+\frac{q+1}{q-1} U\right]^{2}}
$$

where $X=T w, T=\bar{R}^{q / 2} U^{1 / 4}$. Since $B(\rho)=O\left(\rho^{-2}\right)$ it follows from a theorem by Dunkel [25] that there exists local solutions near $\rho=\infty$ of the form

$$
X(\rho)=e^{-\Omega \rho}[1+c(\rho)],
$$

where $\Omega=\sqrt{-\lambda}$ and where $c(\rho)$ is a twice continuously differentiable function which satisfies $c(\rho) \rightarrow 0$ and $\partial_{\rho} c(\rho) \rightarrow 0$ as $\rho \rightarrow \infty$. The introduction of the "tortoise" coordinate $\rho$ is important for the case $q=2$, where

$$
\rho=\sqrt{\bar{R}} \sqrt{\bar{R}-1}+\log (\sqrt{\bar{R}}+\sqrt{\bar{R}-1})
$$

and so $e^{-\Omega \rho}=\bar{R}^{-\Omega / 2} e^{-\Omega \bar{R}}[b+\tilde{c}(\bar{R})]$, where $b=2^{-\Omega} e^{\Omega / 2}$ and $\tilde{c}(\bar{R})$ is twice continuously differentiable and satisfies $\tilde{c}(\bar{R}) \rightarrow 0, \partial_{\bar{R}} \tilde{c}(\bar{R}) \rightarrow 0$ as $\bar{R} \rightarrow \infty$. For $q \geq 3$ one can show that $h=1+O\left(\bar{R}^{-1}\right)$, and in those cases, one can simply replace $e^{-\Omega \rho}$ by $e^{-\Omega \bar{R}}$ in the expression (65). 
Therefore, there exists local solutions $w_{L}, w_{R}$, near left and right boundary points respectively, of the form

$$
\begin{aligned}
& w_{L}(\lambda ; \bar{R})=1-\left(2 \frac{q+1}{q-1}+\frac{\lambda}{(q-1)^{2}}\right) U+O\left(U^{2}\right), \\
& w_{R}(\lambda ; \bar{R})=U^{-1 / 4} \bar{R}^{-q / 2} e^{-\sqrt{-\lambda} \rho}[1+c(\lambda ; \rho)]
\end{aligned}
$$

where $c(\lambda ; \rho)$ is a twice continuously differentiable function which satisfies $c(\lambda ; \rho) \rightarrow 0$ and $\partial_{\rho} c(\lambda ; \rho) \rightarrow 0$ as $\rho \rightarrow \infty$. By varying $\lambda$ in the range of negative real values, we can look for a global solution by the "shooting and matching" procedure.

In what follows, we will prove that there exists such a global solution and that it is unique up to a multiplicative factor. The idea is to use the fact that the local solution at $\bar{R}=1$ for $\lambda=0$, which is given explicitly by

$$
w_{L}(0 ; \bar{R})=w_{0}(\bar{R}) \equiv \frac{q-1-(q+1) U}{q-1+(q+1) U},
$$

has exactly one node in the interval $0<\bar{R}<\infty$ and use the nodal theorem which states that the number of bound states is equal to the number of nodes of the local solution belonging to zero eigenvalue. For a precise statement of this theorem, which also applies to coupled systems of Schrödinger equations, we refer to [26]. Below, we provide an elementary proof for the existence of a unique negative eigenvalue. This proof consists of three parts: (i) To show that the eigenvalues are non-degenerate assuming they exist; (ii) that an eigenvalue exists, by demonstrating that the 'shooting and matching' procedure has a solution and (iii) that the eigenvalue is unique.

In order to proof these assertions we rely on the following observations. Let $w_{1}$ and $w_{2}$ be two local solutions to the eigenvalue problem (60), belonging to the eigenvalues $\lambda_{1}$ and $\lambda_{2}$, respectively. The Wronski determinant is defined by

$$
W\left[w_{1}, w_{2}\right]=U \bar{R}^{q}\left(w_{1}^{\prime} w_{2}-w_{2}^{\prime} w_{1}\right)
$$

and by virtue of Eq. (60), it satisfies

$$
W^{\prime}\left[w_{1}, w_{2}\right]=\left(\lambda_{2}-\lambda_{1}\right) \bar{R}^{q} w_{1} w_{2}
$$

The Wronski determinant will play a central role in proving the three statements above.

(i) The eigenvalues are non-degenerate. Suppose there are two eigenfunctions, $w_{1}$, $w_{2}$ belonging to the same eigenvalue $\lambda_{1}=\lambda_{2}$. Then, Eq. (70) implies that $W\left[w_{1}, w_{2}\right]=$ const. But this constant is zero since $w_{1}$, $w_{2}$ and their derivatives are bounded near $\bar{R}=1$, and because of the factor $U$ in the definition of the Wronski determinant. Therefore, $W\left[w_{1}, w_{2}\right] \equiv 0$, which means that $w_{1}$ and $w_{2}$ are linearly dependent.

(ii) There exists a negative eigenvalue. Let $\bar{R}_{1}>1$ be large enough such that for each $-2\left(q^{2}-1\right)-1 \leq \lambda \leq 0$ and all $\bar{R} \geq \bar{R}_{1}, w_{R}(\lambda ; \bar{R})>0, w_{R}^{\prime}(\lambda ; \bar{R})<0$ and $w_{0}(\bar{R})<0$. That we can always choose such a $\bar{R}_{1}$ follows from the definition of $w_{0}$ and the behavior of $w_{R}$ near the right boundary. Additionally, we can extend $w_{L}(\lambda ; \bar{R})$ to $\bar{R}=\bar{R}_{1}$. Now define $W(\lambda)=\left.W\left[w_{L}(\lambda), w_{R}(\lambda)\right]\right|_{\bar{R}=\bar{R}_{1}}$. We can match $w_{L}(\lambda)$ and $w_{R}(\lambda)$ if and only if $W(\lambda)=0$. So our task it to show that the function $\lambda \mapsto W(\lambda)$, which is continuous, possesses a zero. Now choose $\lambda=\lambda_{1} \equiv-2\left(q^{2}-1\right)-1$. Since

$$
w_{L}\left(\lambda_{1} ; \bar{R}\right)=1+\frac{U}{(q-1)^{2}}+O\left(U^{2}\right)
$$

we have $w_{L}\left(\lambda_{1} ; 1\right)=1, w_{L}^{\prime}\left(\lambda_{1} ; 1\right)>0$. Furthermore, it follows from Eq. 60 that for $\lambda<-2\left(q^{2}-1\right), w(\bar{R})$ cannot have a local maxima as long as $w>0$. Therefore, $w_{L}\left(\lambda_{1} ; \bar{R}\right)$ is monotonously increasing and we must have $w_{L}\left(\lambda_{1} ; \bar{R}_{1}\right)>0$, $w_{L}^{\prime}\left(\lambda_{1} ; \bar{R}_{1}\right)>0$. This implies that $W\left(\lambda_{1}\right)>0$. On the other hand, for $\lambda=0$, we have $w_{L}\left(0 ; \bar{R}_{1}\right)<0, w_{L}^{\prime}\left(0 ; \bar{R}_{1}\right)<0$ which implies $W\left(\lambda_{1}\right)<0$. Therefore, there exists $\lambda$ with $\lambda_{1}<\lambda<0$ for which $W(\lambda)=0$ and for which the local solutions $w_{L}(\lambda)$ and $w_{R}(\lambda)$ can be matched to yield an eigenfunction $w_{\lambda}(\bar{R})$ belonging to the negative eigenvalue $\lambda$.

(iii) The negative eigenvalue is unique. In order to prove this, we first show that $w_{\lambda}(\bar{R})$ possesses no nodes in $(1, \infty)$. We normalize $w_{\lambda}$ such that $w_{\lambda}(1)=1$. Suppose that $w_{\lambda}$ has a node at $\bar{R}_{2}>1$, and that $w_{\lambda}$ is strictly positive on $\left(1, \bar{R}_{2}\right)$. Then, $w_{\lambda}^{\prime}\left(\bar{R}_{2}\right) \leq 0$. Integrating Eq. (170) from $\bar{R}=1$ to $\bar{R}=\bar{R}_{2}$ we have, choosing $\lambda_{1}=\lambda$ and $\lambda_{2}=0$,

$$
\left[U \bar{R}^{q} w_{\lambda}^{\prime} w_{0}\right]_{\bar{R}=\bar{R}_{2}}=W\left[w_{\lambda}, w_{0}\right]\left(\bar{R}_{2}\right)-W\left[w_{\lambda}, w_{0}\right](1)=-\lambda \int_{1}^{\bar{R}_{2}} \bar{R}^{q} w_{\lambda} w_{0} d \bar{R}
$$


It follows that $w_{0}\left(\bar{R}_{2}\right)<0$ for otherwise, the left-hand side would be negative or zero and the right-hand side positive. Therefore, $\bar{R}_{2}$ lies to the right of the single node of $w_{0}$. Next, integrate Eq. (70) from $\bar{R}=\bar{R}_{2}$ to some $\bar{R}>\bar{R}_{2}$ and obtain

$$
U \bar{R}^{q} w_{\lambda}^{\prime}(\bar{R}) w_{0}(\bar{R})=-\lambda \int_{\bar{R}_{2}}^{\bar{R}} \bar{R}^{q} w_{\lambda} w_{0} d \bar{R}+\left[U \bar{R}^{q} w_{\lambda}^{\prime} w_{0}\right]_{\bar{R}=\bar{R}_{2}}+U \bar{R}^{q} w_{\lambda}(\bar{R}) w_{0}^{\prime}(\bar{R})
$$

Suppose first that $w_{\lambda}<0$ on $\left(\bar{R}_{2}, \bar{R}\right)$. Then, the right-hand side is positive, so we must have $w_{\lambda}^{\prime}(\bar{R})<0$. On the other hand, if $w_{\lambda}>0$ on $\left(\bar{R}_{2}, \bar{R}\right)$ the right-hand side is negative, and we must have $w_{\lambda}^{\prime}(\bar{R})>0$. Both options contradict the fact that $w_{\lambda}(\bar{R}) \rightarrow 0$ as $\bar{R} \rightarrow \infty$. Therefore, $w_{\lambda}$ is positive on $(1, \infty)$ and possesses no nodes. Finally, suppose now there exists another eigenfunction $w_{\hat{\lambda}}$ belonging to a negative eigenvalue $\hat{\lambda}>\lambda$. Integrating Eq. (70) over (1, $\left.\infty\right)$ and taking into account the boundary conditions, it follows choosing $\lambda_{1}=\lambda$ and $\lambda_{2}=\hat{\lambda}$ in Eq. (70), that

$$
0=\left.W\left[w_{\lambda}, w_{\hat{\lambda}}\right]\right|_{1} ^{\infty}=(\hat{\lambda}-\lambda) \int_{1}^{\infty} \bar{R}^{q} w_{\lambda} w_{\hat{\lambda}} d \bar{R}
$$

which implies that $w_{\hat{\lambda}}$ must have at least one node. But then we can repeat the argument in the first part of (iii) and reach a contradiction by replacing $w_{\lambda}$ by $w_{\hat{\lambda}}$.

Therefore, there exists a unique non-degenerated negative eigenvalue. Although we have not proven that this eigenvalue corresponds to the ground state, we conjecture that the spectrum of the pulsation operator consists of the single negative eigenvalue and a continuous spectrum $[0, \infty)$.

We have computed the eigenvalue numerically following the "shooting and matching" procedure described in 27]. In order to do so, we rewrote equation (64) in terms of the variable $Y=e^{\Omega \rho} X$ and implemented it numerically. To proceed with the numerical integration we introduce the compactified coordinate $U=1-\bar{R}^{-(q-1)}$ and define left/right boundaries at $U=0+\Delta$ and $U=1-\Delta$ respectively. At these boundaries, initial values are given in correspondence with the local solutions (66), (67), respectively, where the left local solution is truncated after the second order term in $U$ and where $c(\rho)=0$ is chosen in the right solution. The equations are then integrated using the LSODE package 28] combined with a nonlinear Newton iteration to match the solutions at an intermediate point by modifying the parameter $\lambda$ and the scaling of the right solution. The tolerance specified in the LSODE solver, which is used in the variable time-step ODE integrator, was set to TOL $=10^{-13}$. Additionally, we require that at the intermediate point, $Y$ and its derivative match with an error smaller than $10^{-9}$.

As an illustration, figure 3 presents the obtained solutions $Y=e^{\Omega \rho} X$ for the cases $q=2,7,20$. The matching point was chosen at $U=0.6$ but we checked that the obtained results are independent of this choice.

We then use the developed code to obtain the dependence of $\Omega$ on the dimensionality $q$ in the range $q=2,3, \ldots 250$. To ensure convergence of the obtained value we run the code with $\Delta=0.01 / 2^{n}$ ( $n$ an integer number) and increase $n$ until the difference of two successive values of $\Omega$ lies below $0.01 \%$. The results for $\Omega$ are presented in figure 1 while figure 2 displays the percentile error $P E=100|\sqrt{q}-\Omega| / \sqrt{q}$. As expected, the discrepancy diminishes as $q$ increases.

\section{FINAL WORDS}

This work has put on firm grounds the existence of critical phenomena in bubble spacetimes confirming the indications obtained via numerical simulations. We find spherically symmetric, in $z$ homogeneous, linear modes that depart like $\exp \left(\Omega t / R_{0}\right)$ from the critical solution which is static 32 . We have identified the critical solution, the growth rate of the unstable mode and established the universality of the growth rate. The universality follows from the fact that it is possible to decouple the pulsation equations into two Sturm-Liouville problems, where one problem does not give rise to instabilities while the other one is manifestly independent of the background solution when expressed in terms of appropriate dimensionless quantities. Additionally, the study of the unstable Sturm-Liouville problem allows for a derivation of the simple relation $\Omega \simeq q^{1 / 2}$ in the limit of large $q$ 's. This relation agrees very well with the numerical results.

Furthermore, we have made use of a double analytical continuation to map the solution to a stationary mode of the corresponding black strings which is proportional to $e^{i \Omega z / R_{0}}$. This mode represents a static deformation of the black string, with harmonic dependency in $z$. This mode is allowed if the asymptotic periodicity of the extra dimension is an integer multiple of $L_{c}=2 \pi R_{0} / \Omega$. For the uncharged case, the obtained values of $L_{c}$ give the the critical length for the Gregory-Laflamme instability 11, 12]. In the charged case, the black string solutions given by (12) do not correspond to those considered in [12] since we restricted ourselves to a $\mathbf{U}(\mathbf{1})$ gauge field in the absence of a dilaton. 


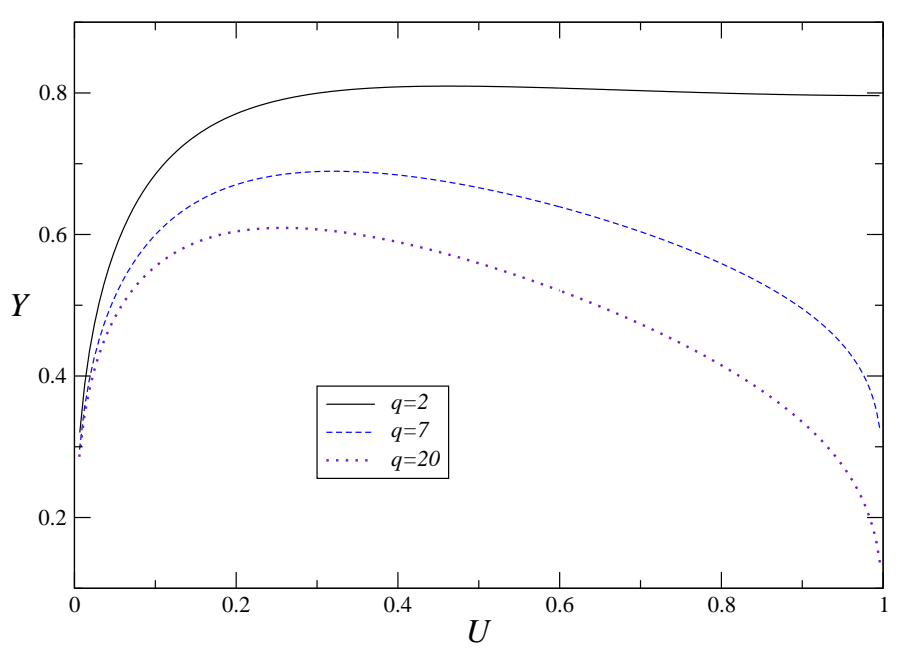

FIG. 3: The eigenfunction $Y=e^{\Omega \rho} X$ as a function of $U$ for three different values of $q$.

We give the dependency for the critical length in terms of the parameter $\epsilon$ in an analytic way. This result can be expressed in terms of the dimensionless mass introduced in [17]

$$
\mu:=\frac{M_{c}}{L_{c}^{q}}=\frac{\left|S^{q}\right|}{16 \pi}\left(\frac{\Omega}{2 \pi}\right)^{q-1}\left[q+2 \epsilon\left(q-\frac{1}{q}\right)\right] .
$$

For large $q$ we can approximate $\Omega \simeq q^{1 / 2}$ and find

$$
\mu \simeq \frac{\sqrt{q}}{16}\left(\frac{e}{2 \pi}\right)^{q / 2}(1+2 \epsilon),
$$

which provides a different way of deriving the law presented in Ref. 17, 20], and generalizes it to the charged case.

Note there is a remarkable difference between the instability of the charged strings considered here and those in [12, 19]. Whereas the addition of charge makes the critical length, at which the instability arises, shorter in our case -making the string "more unstable" - while longer (and the extremal case marginally stable) in 12, 19]. The reason for such a marked different is likely due to the interaction with a magnetic field in 12, 19] while an electric field in the present case. Thus, the dynamics of charged strings even in 5-dimensional cases appears to be quite rich.

Finally, (single) Wick rotations are often used to study and understand phenomena in the Euclidean sector and draw conclusions about black branes. Here, and also in [7], it is show that the understanding of solutions of bubble spacetimes can be used to shed light into black string systems and viceversa.

\section{ACKNOWLEDGMENTS}

We wish to give special thanks to H. Beyer for many useful discussions and for pointing out to us Ref. [25], R. Myers for continuous discussions on bubbles and strings and R. Emparan for several valuable comments and suggestions. We also acknowledge interesting discussions and comments with M. Choptuik, J. Hovdebo, D. Neilsen, F. Pretorius, J. Pullin and J. Ventrella. This work was supported in part by the Center for Computation \& Technology at Louisiana State University, by grants NSF-PHY-0244335, NSF-PHY-0244299, NSF-INT-0204937, NASA-NAG5-13430 and a Research Innovation Award from Research Corporation to Louisiana State University and by funds from the Horace Hearne Jr. Laboratory for Theoretical Physics. We thank the National University of Cordoba and L.L. thanks the Perimeter Institute for Theoretical Physics for hospitality where parts of this work were completed. L.L. is an Alfred P. Sloan Fellow.

[1] E. Witten, Nucl. Phys. B 195, 481 (1982).

[2] D. Brill and H. Pfister, Phys. Lett. B 228, 359 (1989). 
[3] D. Brill and G.T. Horowitz, Phys. Lett. B 262, 437 (1991).

[4] R. Emparan and H. S. Reall, Phys. Rev. D 65, 084025 (2002).

[5] O. Aharony, M. Fabinger, G.T. Horowitz and E. Silverstein, JHEP 0207, 007 (2002).

[6] H. Elvang and G. T. Horowitz, Phys. Rev. D 67, 044015 (2003).

[7] H. Elvang, T. Harmark and N. A. Obers, arXiv:hep-th/0407050

[8] T. Shiromizu, S. Tomizawa, Y. Uchida and S. Mukohyama, arXiv:hep-th/0406181

[9] O. Sarbach and L. Lehner, Phys. Rev. D 69, 021901 (2004).

[10] O. Sarbach and L. Lehner, in preparation.

[11] R. Gregory and R. Laflamme, Phys. Rev. Lett. 70, 2837 (1993).

[12] R. Gregory and R. Laflamme, Nucl. Phys. B 428, 399 (1994).

[13] D. Gross, M.J. Perry and L.G Yaffe. Phys. Rev. D 25, 330 (1982).

[14] C. Gundlach, Phys. Rept. 376, 339 (2003).

[15] R. Gregory and R. Laflamme, Phys. Rev. D 37, 305 (1988).

[16] H. Reall, Phys. Rev. D 64, 044005 (2001).

[17] E. Sorkin, Phys. Rev. Lett. 93, 031601 (2004).

[18] G.T. Horowitz and K. Maeda, Phys. Rev. D 65, 104028 (2002).

[19] R. Gregory and R. Laflamme, Phys. Rev. D 51, 305 (1995).

[20] B. Kol and E. Sorkin, On black-brane instability in an arbitrary dimension, arXiv:gr-qc/0407058

[21] O. Brodbeck, M. Heusler and N. Straumann, Phys. Rev. D 53, 754 (1996).

[22] M. Reed and B. Simon, Methods of modern mathematical physics, Volume II, Academic Press (1980).

[23] See for example K. Jörgens and F. Rellich, Eigenwerttheorie gewöhnlicher Differentialgleichungen, Springer-Verlag, New York 1976.

[24] see, for instance, W. Walter, Ordinary Differential Equations (Springer, New York 1998).

[25] O. Dunkel, Am. Acad. Arts Sci. Proc. 38, 341 (1912).

[26] H. Amann and P. Quittner, J. Math. Phys. 36, 4553 (1995).

[27] W. Press, S. Teukoksly, W. Vetterling and B. Flannery Numerical Recipes, Cambridge University Press, New York (1992).

[28] http://www.llnl.gov/CASC/odepack/download/lsode-agree.html

[29] J Hovdebo, private communication (2004).

[30] For a review on critical phenomena in G.R. see 14]

[31] see, for instance, Ref. [21] for a derivation in the case $q=2$.

[32] Recently, an independent analysis, also styding gauge-independent linearized perturbations off the critical solution for the case $q=2$ has obtained values in full agreement with the ones presented here [29]). 